\title{
Retinal and choroidal expression of BMP-2 in lens-induced myopia and recovery from myopia in guinea pigs
}

\author{
HONGHUI LI, JUAN WU, DONGMEI CUI and JUNWEN ZENG \\ State Key Laboratory of Ophthalmology, Zhongshan Ophthalmic Center, \\ Sun Yat-Sen University, Guangzhou, Guangdong 510060, P.R. China
}

Received March 11, 2015; Accepted January 8, 2016

DOI: $10.3892 / \mathrm{mmr} .2016 .4843$

\begin{abstract}
The present study investigated the retinal and choroidal expression of bone morphogenetic protein-2 (BMP-2) in myopia and in myopia recovery in a guinea pig model. For this investigation, two groups of guinea pigs, lens-induced myopia and recovery from myopia, were used, and defocused myopia was induced the guinea pigs wearing $-4.00 \mathrm{D}$ lenses on the right eyes for 3 weeks, with the left eyes serving as the contralateral. In the following week, the lenses of the guinea pigs in the recovery group were removed, and the refractive power and axial length were measured. The expression of BMP-2 in the eyeballs was observed using immunohistochemistry and analyzed using Western blot analysis. After 3 weeks, the eyes acquired relative myopia and longer axial lengths in the two groups of guinea pigs. After 1 week without lenses in the recovery group, the myopia and axial lengths regressed. Immunofluorescence staining showed that BMP-2 was expressed in the posterior retina, RPE, choroid and sclera. The expression of BMP-2 decreased in the myopic retina of the guinea pigs. Following the regression of myopia in the recovery group, no difference in the expression of BMP-2 was observed between the recovered treated eyes and the contralateral eyes. The choroidal expression level of BMP-2 in the treated eyes showed no significant changes in either group. Therefore, BMP-2 may be involved in the development of myopia, however, it does not have a primary role in the retinal and choroidal signals regulating scleral remodeling.
\end{abstract}

Correspondence to: Professor Junwen Zeng, State Key Laboratory of Ophthalmology, Zhongshan Ophthalmic Center, Sun Yat-Set University, 54 South Xianlie Road, Guangzhou, Guangdong 510060, P.R. China

E-mail: cier72@163.com

Key words: bone morphogenetic protein-2, myopia, retina, choroid, guinea pig

\section{Introduction}

Myopia is a prevalent ocular disorder, which is accompanied by the potentially blinding complications of high myopia and the primary structural cause of which is increased axial length of the eye (1). Eye growth and refractive development are regulated by local retinal optical signal in chicks, tree shrews and monkeys (2). A blurred image on the retina initiates a signaling cascade, which begins in the retinal amacrine cells (3), and traverses the choroid (4) to alter extracellular matrix remodeling in the sclera (5), thus facilitating accelerated eye growth and, in the longer term, changes in eye size. This signaling pathway within the eye, comprising a retina to sclera cascade, affects retinal pigment epithelium (RPE) physiology (6) and choroidal thickness (7), thus contributing to ocular refractive change in the short-term.

Several studies have focused on identifying the components of the retinal and choroidal signaling cascade responsible for the transduction of the blur signal (8), and the transmission of this information from these tissues to the sclera (9). Several factors have been confirmed to be involved in this process, including retinoic acid (4), transforming growth factor (TGF) $\beta$ and basic fibroblast growth factor (bFGF) $(10,11)$.

Bone morphogenetic proteins (BMPs) are the largest subfamily of the TGF- $\beta$ family (12), and several ocular tissues, including the cornea, lens, RPE and retina express BMPs (13). BMP-2 belongs to the BMPs family and is an important factor for early eye morphogenesis, ocular development and growth (14-16). The involvement of BMP-2 in retinal and choroidal signaling during myopia has been previously investigated. In form-deprived myopia of guinea pigs, the scleral expression of BMP-2 decreased (17). In vitro, BMP-2 promotes scleral fibroblast proliferation and extracellular matrix synthesis $(18,19)$. This suggests that BMP-2 may be involved in scleral remodeling in myopia development. The genetic analysis of humans has confirmed that BMP-2 is associated with refractive error and myopia $(20,21)$. However, whether BMP2 affects ocular growth via the choroid and retina or by directly acting on the sclera remains unknown.

The aim of the present study was to characterise the contribution of retinal and choroidal BMP-2 to the retinoscleral cascade in a mammalian model of myopia development. BMP-2 expression levels changes in the retina and choroid were analyzed during myopia development and recovery from myopia. 


\section{Materials and methods}

Lens-induced myopia model preparation. A total of 24 pigmented guinea pigs, aged 3 weeks and weighing $180-220 \mathrm{~g}$, were provided by Shandong Traditional Chinese Medicine University (Shandong, China). Animals were maintained in an air-conditioned room with an ambient temperature of $16-26^{\circ} \mathrm{C}$, 40-70\% relative humidity and 12-h light/dark cycle (daytime light intensity, 500 lux). Animals had ad libitum access to water supplemented with vitamin $\mathrm{C}$ and food (guinea pig pellets, hay and fresh vegetables). All the guinea pigs were fed indoors in a standardized manner. In all guinea pigs defocused myopia was induced by the animals wearing a concave lens (-4.00 D; polymethyl methacrylate; King Tak \& Jia Run Co., Beijing, China) in the right eye, with the left eye serving as a control. The animals were randomly assigned to either the lens-induced myopia (LIM) group, in which animals wore lenses for 3 weeks, and a recovery group, in which animals wore lenses for 3 weeks, followed by 1 week without the lenses. Each group contained 12 guinea pigs. During the experimental period, the lenses were cleaned once daily in order to prevent the form-deprival effect (22). Animals were treated three times with local cycloplegia in both eyes in order to produce mydriasis and cycloplegia and, 30 mins after completion of the three treatments, the animals were examined using cycloplegic streak retinoscopy (66 Vision-Tech Co., Ltd., Suzhou, China). The axial length was measured by A-ultrasonic scanning with a 10-MHz probe (KN-1800; Kangning Medical Device Co., Ltd., Wuxi, China) every week.

All animals were treated according to the Association for Research in Vision and Ophthalmology statement for the Use of Animals in Ophthalmic and Vision Research, and the present study was approved by the Institutional Animal Care and Use Committee of Zhongshan Ophthalmic Center of Sun Yat-Sen University (Guangzhou, China).

Immunofluorescent microscopy. Following the final measurements of axial length and refractive power (LIM group, 3 weeks; recovery group, 4 weeks), the guinea pigs were sacrificed via an intraperitoneal injection of $10 \%$ chloral hydrate (200 mg/kg; Boster Biological Technology Co., Ltd., Wuhan, China). Their eyes were rapidly enucleated and then fixed in $4 \%$ neutral-buffered formalin (Boster Biological Technology Co., Ltd.) for $24 \mathrm{~h}$ at $4^{\circ} \mathrm{C}$. The tissue was dehydrated and cut into frozen sections ( $5 \mu \mathrm{m}$ thick), with the posterior eye ball around the optic nerve selected for analysis. The samples were blocked with normal serum (Boster Biological Technology Co., Ltd.) for $30 \mathrm{~min}$ at room temperature, followed by incubation with rabbit anti-BMP-2 polyclonal antibody (1:500; ab82511; Abcam, Cambridge, MA, USA) at $4^{\circ} \mathrm{C}$ overnight and incubation with fluorescein isothiocyanate-conjugated goat anti-rabbit IgG antibodies (1:50; BA1105; Boster Biological Technology Co., Ltd.) for $1 \mathrm{~h}$ at room temperature. Finally, the nuclei were stained with 4,6-diamidino-2-phenylindole (Roche Diagnostics, Indianapolis, IN, USA) for $15 \mathrm{~min}$, and the slides were mounted. The samples were observed under a confocal fluorescent microscope (LSM 510 META; Carl Zeiss, Inc., Oberkochen, Germany).

Western blot analysis. The eyes of the animals were rapidly enucleated and hemisected. The posterior neural retina was dissected with minimal adherent retinal pigment epithelium, and the choroid was then removed from the underlying sclera. The tissues were collected and frozen in liquid nitrogen. Each frozen tissue sample was ground, and the total protein was collected using lysis buffer with $1 \%$ phenylmethanesulfonyl fluoride (Beyotime Institute of Biotechnology, Shanghai, China). The protein concentration was assayed using a Bicinchoninic Acid Protein Assay kit (Beyotime Institute of Biotechnology). Subsequently, $30 \mu \mathrm{g}$ of the protein samples were separated by 10\% SDS-PAGE (Boster Biological Technology Co., Ltd.) and electrotransferred onto polyvinylidene fluoride membranes (EMD Millipore, Billerica, MA, USA) at $200 \mathrm{~mA}$ for $2 \mathrm{~h}$, which were then blocked with $5 \%$ bovine serum albumin (Sigma-Aldrich, St.Louis,MO, USA) for $2 \mathrm{~h}$ at room temperature. The membranes were then incubated with mouse anti- $\beta$-actin monoclonal antibody (1:3,000; ab8226; Abcam) and rabbit anti-BMP-2 polyclonal antibody (1:500; ab82511), respectively, overnight at $4^{\circ} \mathrm{C}$. The membranes were then washed three times with Tris-buffered saline with Tween-20 (TBST) and incubated with horseradish peroxidase (HRP)-conjugated goat anti-mouse (1:2,000; BA1050) and goat anti-rabbit $(1,2000 ;$ BA1054; both Boster Biological Technology) IgG secondary antibodies for $1 \mathrm{~h}$ at room temperature. Following washing three times with TBST, the proteins were detected using an enhanced chemiluminescence detection system (EMD Millipore, Billerica, MA, CA), exposed onto negative film, developed and fixed. The film was scanned and subsequently analyzed using Quantity One Imaging Software (version 1; Bio-Rad Laboratories, Inc., Hercules, CA, USA). $\beta$-actin served as an internal reference, and the experiments were repeated at least three times.

Statistical analysis. Each experiment was repeated at least three times. Data are expressed as the mean \pm standard error of the mean. The measurements of refractive power and axial lengths in each group of guinea pigs were analyzed using a paired sample t-test. The statistical significance of differences were determined by one-way analysis of variance followed by Tukey's post-hoc multiple comparison test, using a Mann-Whitney U test when significance was detected. All data analysis was performed using SPSS version 19 (SPSS, Inc, Chicago, IL, USA). P $<0.05$ was considered to indicate a statistically significant difference.

\section{Results}

Refractive power and axial lengths of guinea pigs. No significant differences in refraction power or axial length were found between the treated and untreated eyes of the two groups of guinea pigs prior to myopia induction $(\mathrm{P}>0.05)$. Following treatment to induce myopia using minus lenses for 3 weeks, the treated eyes in the two groups developed myopia $(\mathrm{P}<0.05 ;$ Fig. $1 \mathrm{~A})$, and had longer axial lengths $(\mathrm{P}<0.05$; Fig. $1 \mathrm{~B})$, compared with the contralateral eyes. In the recovery group, without lenses for 1 week, the changes in the refractive power and axial lengths of the treated eyes had regressed, and there were no differences in refraction $(\mathrm{P}>0.05)$ or axial length $(\mathrm{P}>0.05)$ between the treated and untreated eyes.

Expression of BMP-2 in the eyes of the LIM and myopia recovery groups of guinea pigs. Immunofluorescence 
A

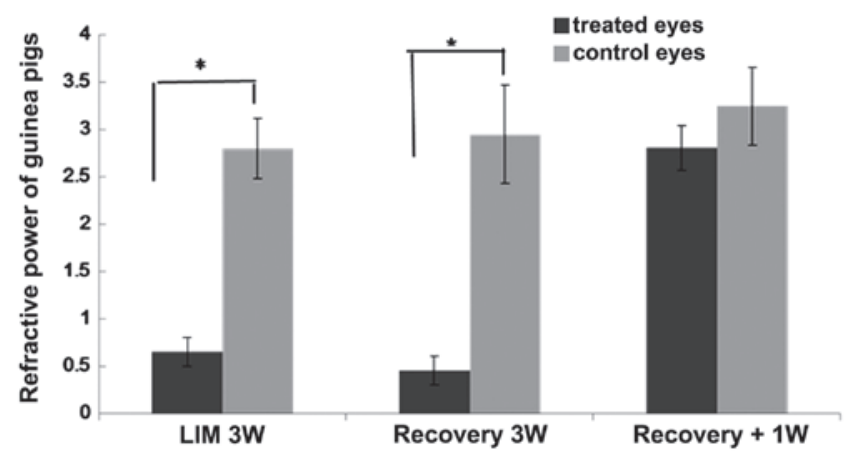

B

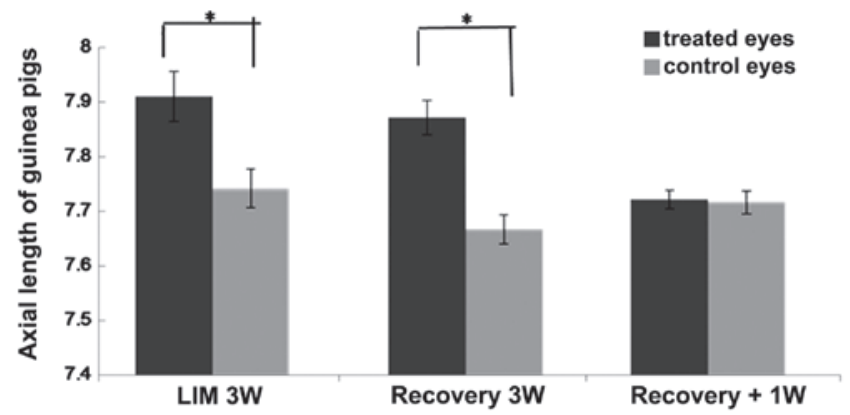

Figure 1. (A) Refractive power and (B) axial lengths of guinea pig eyes. Following the wearing lenses for 3 weeks, the treated eyes of the guinea pigs in the LIM and recovery groups developed myopia and acquired elongated axial lengths, compared with the contralateral eyes $(\mathrm{P}<0.05)$. In the recovery group, 1 week without lenses led to regression of the myopia and axial lengthening. No differences in refraction or axial length were found between the treated and control eyes $(\mathrm{P}>0.05)$. Data are presented as the mean \pm standard error of the mean $(\mathrm{n}=3)$. ${ }^{*} \mathrm{P}<0.05$, vs. control. LIM, lens-induced myopia; $3 \mathrm{~W}, 3$ weeks; $1 \mathrm{~W}, 1$ week.

A

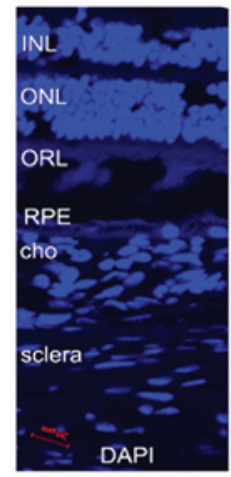

C

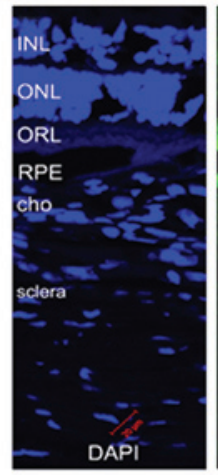

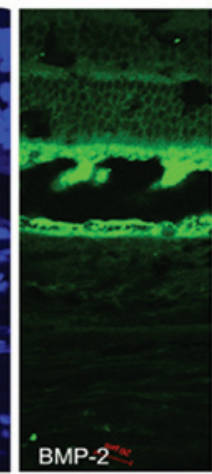
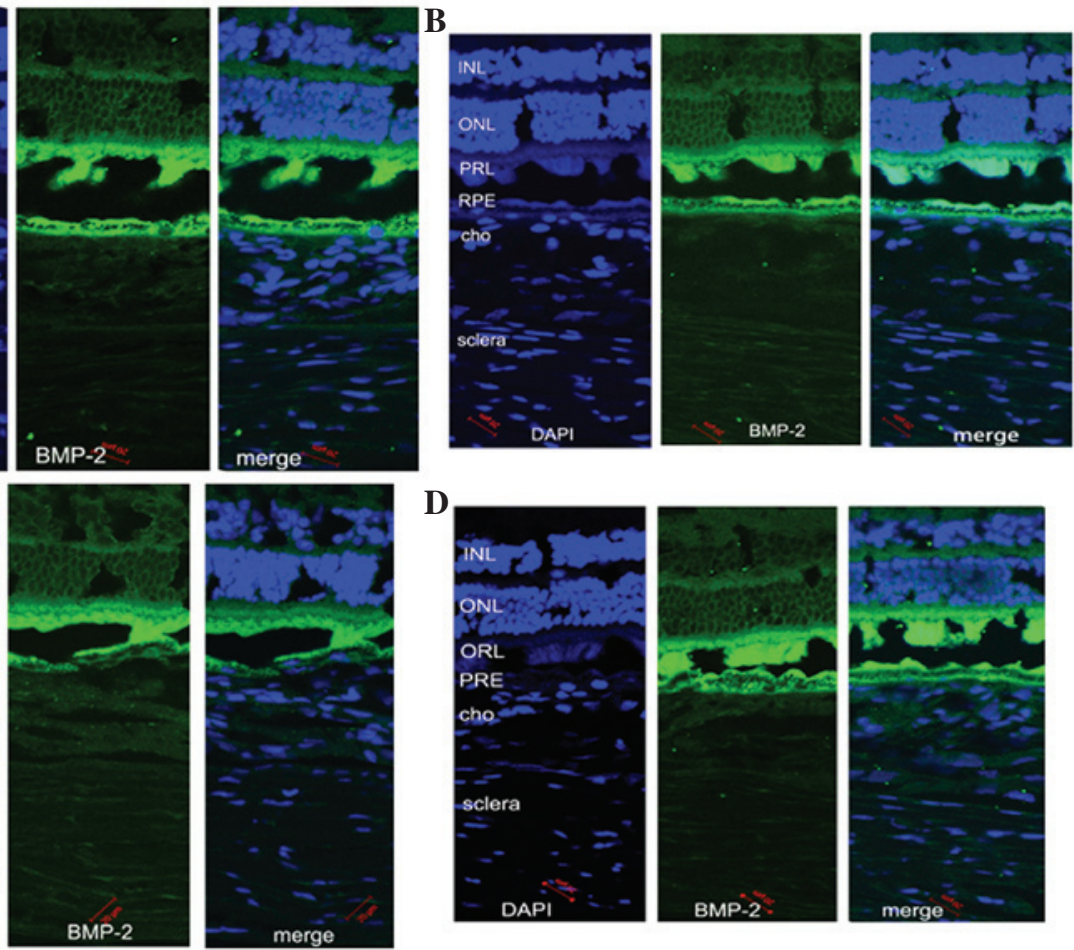

Figure 2. Immunoreactivity for BMP-2 (green) antibody and DAPI (blue) in the retina, RPE, cho and sclera of guinea pig eyes. Compared with the (A) untreated eyes, BMP-2 antibody staining in the retina of the (B) myopic eyes was weaker. In the recovery group, in which myopic eyes were without lenses for 1 week, BMP-2 antibody staining in the retina of the (C) treated eyes and (D) untreated eyes were similar. Images were captured using a confocal microscope (magnification, x200; scale bar, $20 \mu \mathrm{m}$ ). BMP-2, bone morphogenetic protein-2; INL, inner nuclear layer; ONL, outer nuclear layer; ORL, optical receptor layer; RPE, retinal pigment epithelium; cho choroid; DAPI, 4,6-diamidino-2-phenylindole.

staining was used to examine the expression levels of BMP-2 in the posterior retina, RPE, choroid and sclera of the guinea pig eyes (Fig. 2). In the LIM group, the protein expression of BMP-2 in the myopic retina appeared weaker, compared 


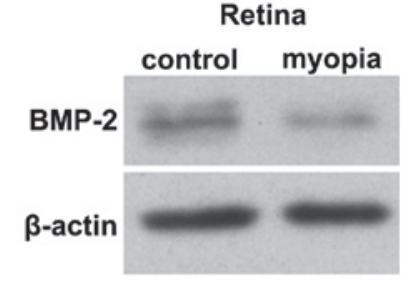

B

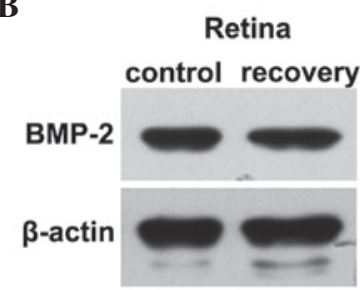

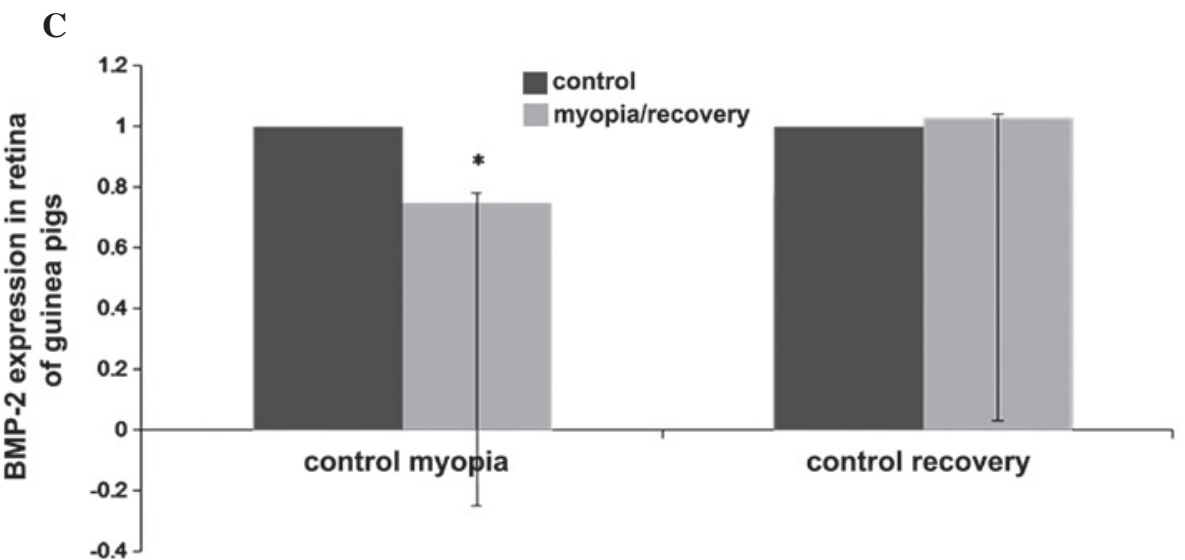

Figure 3. Protein expression of BMP-2 in the retina of guinea pig eyes. (A) In the myopic eyes of the lens-induced myopia group, the retinal level of BMP-2 decreased, compared with the that in the control eyes $(\mathrm{P}<0.05)$. (B) When myopia and axial lengths regressed in the recovery group, no differences were observed between the retinal level of BMP-2 between the treated and untreated eyes $(\mathrm{P}>0.05)(\mathrm{C})$ Data are presented as the mean \pm standard error of the mean $(\mathrm{n}=3) .{ }^{*} \mathrm{P}<0.05$, vs. control. BMP-2, bone morphogenetic protein-2.

A

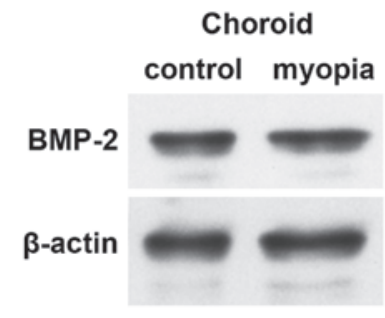

C

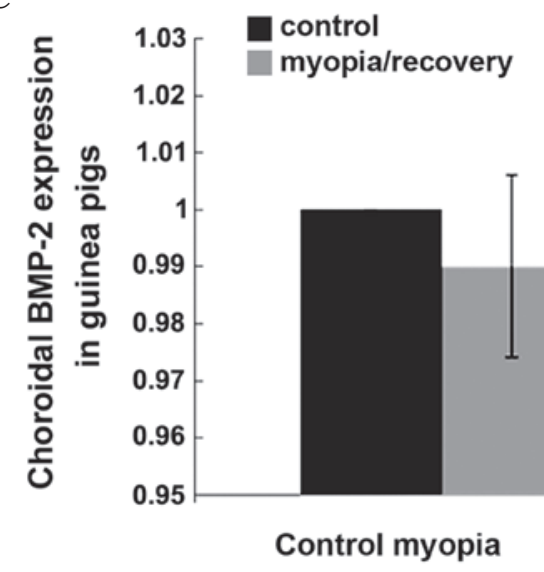

B

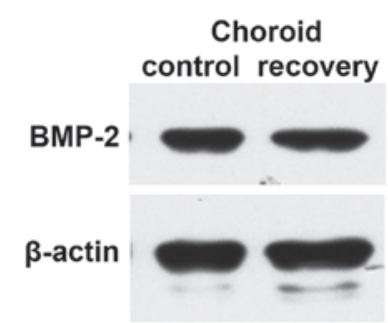

Figure 4. Protein expression levels of BMP-2 in the choroid of guinea pig eyes. (A) In the lens-induced myopia group, no significant difference was observed in the choroidal expression of BMP-2 in the myopic eyes, compared with the untreated control eyes $(\mathrm{P}>0.05)$. Similarly, in the $(\mathrm{B})$ recovery group, in which myopia and axial lengths regressed, no significant difference was observed in the choroidal expression of BMP-2 between the treated and untreated control eyes $(\mathrm{P}>0.05)$. (C) Data are presented as the mean \pm standard error of the mean $(\mathrm{n}=3)$. ${ }^{*} \mathrm{P}<0.05$, vs. control. BMP-2, bone morphogenetic protein-2.

with that in the control eyes (Fig. 2A and B). However, following recovery from myopia, no significant differences were observed in the expression of BMP-2 in the treated and untreated control eyes (Fig. 2C and D).
Retinal expression of BMP-2 in the LIM and myopia recovery groups of guinea pigs. Western blot analysis was used to further verify the changes in the protein expression levels of BMP-2 observed in the posterior retina and choroid of the guinea pigs. 
Following 3 weeks of lens wearing in the LIM group, the retinal protein levels of BMP-2 in the myopic eyes were decreased significantly, compared with levels in the contralateral eyes $(\mathrm{P}<0.05$; Fig. 3A). In the recovery group, following 1 week without lenses subsequent to 3 weeks wearing lenses, no significant differences were observed in the protein levels of BMP-2 between the treated eyes and the contralateral untreated eyes ( $\mathrm{P}>0.05$; Fig. 3B).

Choroidal expression of BMP-2 in the LIM and myopia recovery groups of guinea pigs. Following 3 weeks of lens wearing in the LIM group, no differences were observed between the choroidal protein levels of BMP-2 in the treated eyes and those in the contralateral untreated eyes ( $\mathrm{P}>0.05$; Fig. 4A). Following a further 1 week without lenses, no differences in the choroidal protein expression of BMP-2 were observed between the treated and untreated eyes of the guinea pigs in the recovery group ( $\mathrm{P}>0.05$; Fig. 4B).

\section{Discussion}

Alterations in the expression of BMP-2 are associated with scleral remodeling during myopia $(17,23)$, however, whether the expression of BMP-2 in the retina and choroid is involved in the retinoscleral cascade remains to be fully elucidated. The present study demonstrated that the retinal expression of BMP-2 decreased in the development of myopia, and that its level increased on recovery from myopia. In the choroid, no changes in the expression of BMP-2 were observed during myopia development or on recovery from myopia.

Ocular growth and refractive error have been regulated by inducing hyperopic defocus with negative power lenses, or myopic defocus with positive power lenses, in several animal models, including primates (24-27). Studies have reported that eye growth is regulated by retinal mechanisms, which act locally within the eye, producing a cascade of changes that ultimately affect the structure of the sclera $(28,29)$.

BMP-2 is one of the most widely investigated growth factors in the BMP family and it is essential for the development and patterning of the retina (30). In addition, BMP signaling is neuroprotective for retinal ganglion cells following damage (31) and is involved in glial proliferation (32). BMP-2 may act as a negative growth regulator in the retina and the RPE (33). In the development of myopia, a decrease in the level of BMP-2 was observed in the retina, whereas its level increased following recovery from myopia. As this alteration occurred following significant structural change, it is likely that the retinal level of BMP-2 is associated with eye growth and myopia development. A previous study reported that the retinal gene expression of BMP2 was downregulated in form-deprived myopia in chickens (34). The potential involvement of BMP-2 in the signaling cascade, which mediates the development of myopia remains to be elucidated. During lense-induced myopia development in tree shrews, choroidal gene expression of BMP2 is upregulated, compared with control animals $(35,36)$, and this change may have been part of a common choroidal response. However, in the present study, the choroidal expression of BMP-2 in the guinea pig myopia model remained unchanged.

TGF- $\beta$ is important in myopia. During the development of myopia in tree shrews, the expression levels of three TGF- $\beta$ isoforms (TGF- $\beta 1, \mathrm{TGF}-\beta 2$ and TGF- $\beta 3$ ) in the sclera decrease, and in vitro, TGF- $\beta$ regulates collagen production in the sclera (37). However, the levels of these three isoforms remain within normal ranges in retinal and choroidal tissues during myopia development (38). bFGF is another important regulator in the development of myopia. In the development of myopia, the expression of bFGF in the sclera decreases significantly $(39,40)$, whereas its content and concentration remain unchanged in the retina, RPE and choroid (40). The ocular injection of bFGF inhibits myopia development $(41,42)$, however, whether bFGF acts on the sclera or upstream of retina, RPE or choroid remains to be elucidated. A previous study demonstrated that the intravitreal injection of bFGF acts either parallel to or downstream of the dopaminergic amacrine cells, rather than through them, to prevent myopia development (41), and bFGF intraocular injection suppresses retinal neuronal apoptosis in myopia (42). The peribulbar injection of bFGF also regulates scleral remodeling to prevent myopia in guinea pigs (43). Therefore, the present study hypothesized that, although TGF- $\beta$ and bFGF were involved in scleral regulation during myopia development, they did not have a primary role in the retinal or choroidal signals.

Although a decrease in the retinal expression of BMP-2 was observed in myopia development in the present study, its functional correlation remains to be elucidated. As the retinal and choroidal components of the retinoscleral signal are unlikely to rely upon BMP-2 to modulate downstream scleral remodeling, the signaling cascade relies on multiple signaling pathways to control eye growth in myopia. The change in retinal expression of BMP-2 may regulate cellular responses in pathways other than the choroid to sclera pathway, which results in scleral remodeling and facilitates eye growth (44). In the RPE layer, the gene expression of BMP2 is upregulated in eyes treated with minus lenses, and is downregulated in eyes treated with plus lenses (45). Certain studies have shown that the levels of the three forms of TGF- $\beta$ in the RPE-choroid complex mice with form-deprived myopia are significantly elevated (46). In addition, TGF- $\beta 2$ content and concentration are significantly higher in myopic eyes, in the retina-RPE-choroid and the sclera, in chicks (11). The possible explanation for role of the RPE-choroid complex in myopia development may be due to RPE cells, possessing the ability to express and secrete TGF- $\beta$ (46), and affect choroidal function or tranverse the choroid to affect the sclera.

The results of the present study demonstrated that the retinal expression of BMP2 in the eyes of lens-induced myopia were downregulated, and this expression was upregulated following recovery of the myopic eyes in guinea pigs. This suggested that BMP-2 is involved in the development of myopia in mammals. However, the protein level of BMP-2 in the choroid remained unchanged in the myopic eyes, which suggested that BMP-2 has different roles in the retina and sclera during the development of myopia.

\section{Acknowledgements}

This study was supported by the Province Science Foundation of China (grant no. 10251008901000025). 


\section{References}

1. Li HH, Huo LJ, Gao ZY, Zhao F and Zeng JW: Regulation of scleral fibroblast differentiation by bone morphogenetic protein-2. Int J Ophthalmol 7: 152-156, 2014.

2. Feldkaemper M and Schaeffel F: An updated view on the role of dopamine in myopia. Exp Eye Res 114: 106-119, 2013.

3. Fischer AJ, McGuire JJ, Schaeffel F and Stell WK: Light- and focus-dependent expression of the transcription factor ZENK in the chick retina. Nat Neurosci 2: 706-712, 1999.

4. Mertz JR and Wallman J: Choroidal retinoic acid synthesis: A possible mediator between refractive error and compensatory eye growth. Exp Eye Res 70: 519-527, 2000

5. McBrien NA, Lawlor P and Gentle A: Scleral remodeling during the development of and recovery from axial myopia in the tree shrew. Invest Ophth Vis Sci 41: 3713-3719, 2000.

6. Rymer J and Wildsoet CF: The role of the retinal pigment epithelium in eye growth regulation and myopia: A review. Vis Neurosci 22: 251-261, 2005.

7. Wildsoet $\mathrm{C}$ and Wallman J: Choroidal and scleral mechanisms of compensation for spectacle lenses in chicks. Vision Res 35: 1175-1194, 1995.

8. McBrien NA: Regulation of scleral metabolism in myopia and the role of transforming growth factor-beta. Exp Eye Res 114: 128-140, 2013.

9. Wallman $\mathrm{J}$ and Winawer $\mathrm{J}$ : Homeostasis of eye growth and the question of myopia. Neuron 43: 447-468, 2004.

10. Rohrer B and Stell WK: Basic fibroblast growth factor (bFGF) and transforming growth factor beta (TGF-beta) act as stop and go signals to modulate postnatal ocular growth in the chick. Exp Eye Res 58: 553-561, 1994.

11. Seko Y, Shimokawa H and Tokoro T: Expression of bFGF and TGF-beta 2 in experimental myopia in chicks. Invest Ophthalmol Vis Sci 36: 1183-1187, 1995.

12. Wozney JM: The bone morphogenetic protein family and osteogenesis. Mol Reprod Dev 32: 160-167, 1992.

13. Wordinger RJ and Clark AF: Bone morphogenetic proteins and their receptors in the eye. Exp Biol Med (Maywood) 232: 979-992, 2007.

14. McGlinn AM, Baldwin DA, Tobias JW, Budak MT, Khurana TS and Stone RA: Form-deprivation myopia in chick induces limited changes in retinal gene expression. Invest Ophthalmol Vis Sci 48: 3430-3436, 2007.

15. Sakuta H, Takahashi H, Shintani T, Etani K, Aoshima A and Noda M: Role of bone morphogenic protein 2 in retinal patterning and retinotectal projection. J Neurosci 26: 10868-10878, 2006.

16. Belecky-Adams T and Adler R: Developmental expression patterns of bone morphogenetic proteins, receptors, and binding proteins in the chick retina. J Comp Neurol 430: 562-572, 2001.

17. Wang Q, Zhao G, Xing S, Zhang L and Yang X: Role of bone morphogenetic proteins in form-deprivation myopia sclera. Mol Vis 17: 647-657, 2011.

18. Wang Q, Zhao G, Xing S, Zhang L and Yang X: Role of bone morphogenetic proteins in form-deprivation myopia sclera. Mol Vis 17: 647-657, 2011.

19. Hu J, Cui D, Yang X, Wang S, Hu S, Li C and Zeng J: Bone morphogenetic protein-2: A potential regulator in scleral remodeling. Mol Vis 14: 2373-2380, 2008

20. Yoshikawa M, Yamashiro K, Miyake M, Oishi M, Akagi-Kurashige Y, Kumagai K, Nakata I, Nakanishi H, Oishi A, Gotoh N, et al: Comprehensive replication of the relationship between myopia-related genes and refractive errors in a large Japanese cohort. Invest Ophthalmol Vis Sci 55: 7343-7354, 2014.

21. Verhoeven VJ, Hysi PG, Wojciechowski R, Fan Q, Guggenheim JA, Höhn R, MacGregor S, Hewitt AW, Nag A, Cheng CY, et al: Genome-wide meta-analyses of multiancestry cohorts identify multiple new susceptibility loci for refractive error and myopia. Nat Genet 45: 314-318, 2013.

22. Chen B, Wang C, Chen W and Ma J: Altered TGF- $\beta 2$ and bFGF expression in scleral desmocytes from an experimentally-induced myopia guinea pig model. Graefes Arch Clin Exp Ophthalmol 251: $1133-1144,2013$.

23. Hu J, Cui D, Yang X, Wang S, Hu S, Li C and Zeng J: Bone morphogenetic protein-2: A potential regulator in scleral remodeling. Mol Vis 14: 2373-2380, 2008

24. Benavente-Perez A, Nour A and Troilo D: The effect of simultaneous negative and positive defocus on eye growth and development of refractive state in marmosets. Invest Ophthalmol Vis Sci 53 $6479-6487,2012$
25. Graham B and Judge SJ: The effects of spectacle wear in infancy on eye growth and refractive error in the marmoset (Callithrix jacchus). Vision Res 39: 189-206, 1999.

26. Norton TT and Siegwart JT Jr: Animal models of emmetropization: Matching axial length to the focal plane. J Am Optom Assoc 66: 405-414, 1995.

27. Hung LF, Crawford ML and Smith EL: Spectacle lenses alter eye growth and the refractive status of young monkeys. Nat Med 1: 761-765, 1995.

28. Benavente-Pérez A, Nour A and Troilo D: Axial eye growth and refractive error development can be modified by exposing the peripheral retina to relative myopic or hyperopic defocus. Invest Ophthalmol Vis Sci 55: 6765-6773, 2014.

29. Ashby RS, Zeng G, Leotta AJ, Tse DY and McFadden SA: Egr-1 mRNA expression is a marker for the direction of mammalian ocular growth. Invest Ophthalmol Vis Sci 55: 5911-5921, 2014.

30. Sakuta H, Takahashi H, Shintani T, Etani K, Aoshima A and Noda M: Role of bone morphogenic protein 2 in retinal patterning and retinotectal projection. J Neurosci 26: 10868-10878, 2006.

31. Ueki Y and Reh TA: Activation of BMP-Smad1/5/8 signaling promotes survival of retinal ganglion cells after damage in vivo. PLoS One 7: e38690, 2012

32. Ueki Y and Reh TA: EGF stimulates müller glial proliferation via a BMP-dependent mechanism. Glia 61: 778-789, 2013.

33. Mathura JR Jr, Jafari N, Chang JT, Hackett SF, Wahlin KJ, Della NG, Okamoto N, Zack DJ and Campochiaro PA: Bone morphogenetic proteins-2 and -4: Negative growth regulators in adult retinal pigmented epithelium. Invest Ophthalmol Vis Sci 41: 592-600, 2000

34. McGlinn AM, Baldwin DA, Tobias JW, Budak MT, Khurana TS and Stone RA: Form-deprivation myopia in chick induces limited changes in retinal gene expression. Invest Ophthalmol Vis Sci 48: 3430-3436, 2007.

35. He L, Frost MR, Siegwart JT Jr and Norton TT: Gene expression signatures in tree shrew choroid during lens-induced myopia and recovery. Exp Eye Res 123: 56-71, 2014

36. He L, Frost MR, Siegwart JT Jr and Norton TT: Gene expression signatures in tree shrew choroid in response to three myopiagenic conditions. Vision Res 102: 52-63, 2014.

37. Jobling AI, Nguyen M, Gentle A and McBrien NA Isoform-specific changes in scleral transforming growth factor-expression and the regulation of collagen synthesis during myopia progression. J Biol Chem 279: 18121-18126, 2004.

38. Jobling AI, Wan R, Gentle A, Bui BV and McBrien NA: Retinal and choroidal TGF- $\beta$ in the tree shrew model of myopia: Isoform expression, activation and effects on function. Exp Eye Res 88: 458-466, 2009

39. Chen BY, Wang CY, Chen WY and Ma JX: Altered TGF- $\beta 2$ and bFGF expression in scleral desmocytes from an experimentally-induced myopia guinea pig model. Graefe's Arch Clin Exp Ophthalmol 251: 1133-1144, 2013

40. Seko Y, Shimokawa $\mathrm{H}$ and Tokoro T: Expression of bFGF and TGF-beta 2 in experimental myopia in chicks. Invest Ophthalmol Vis Sci 36: 1183-1187, 1995.

41. Rohrer B, Iuvone PM and Stell WK: Stimulation of dopaminergic amacrine cells by stroboscopic illumination or fibroblast growth factor (bFGF, FGF-2) injections: Possible roles in prevention of form-deprivation myopia in the chick. Brain Res 686: 169-181, 1995.

42. Mao J, Liu S, Wen D, Tan X and Fu C: Basic fibroblast growth factor suppresses retinal neuronal apoptosis in form-deprivation myopia in chicks. Curr Eye Res 31: 983-987, 2006.

43. Tian XD, Cheng YX, Liu GB, Guo SF, Fan CL, Zhan LH and $\mathrm{Xu}$ YC: Expressions of type I collagen, $\alpha 2$ integrin and $\beta 1$ integrin in sclera of guinea pig with defocus myopia and inhibitory effects of bFGF on the formation of myopia. Int J Ophthalmol 6: 54-58, 2013.

44. McBrien NA: Regulation of scleral metabolism in myopia and the role of transforming growth factor-beta. Exp Eye Res 114: 128-140, 2013.

45. Zhang Y, Liu Y and Wildsoet CF: Bidirectional, optical sign-dependent regulation of BMP2 gene expression in chick retinal pigment epithelium. Invest Ophthalmol Vis Sci 53: 6072-6080, 2012

46. Wu PC, Tsai CL, Gordon GM, Jeong S, Itakura T, Patel N, Shi S and Fini ME: Chondrogenesis in scleral stem/progenitor cells and its association with form-deprived myopia in mice. Mol Vis 21: 138-147, 2015 . 